
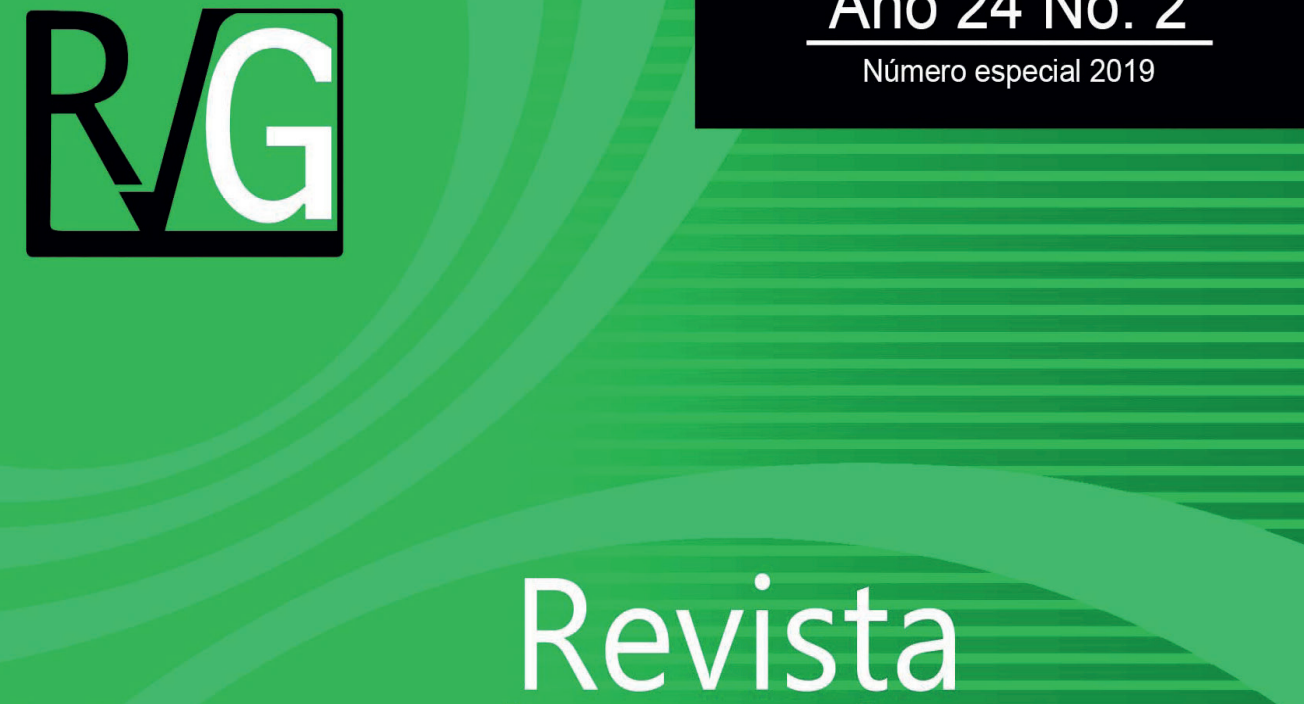

Venezolana de

$\frac{1}{6}$

$\frac{\mathbb{O}}{\frac{1}{0}}$
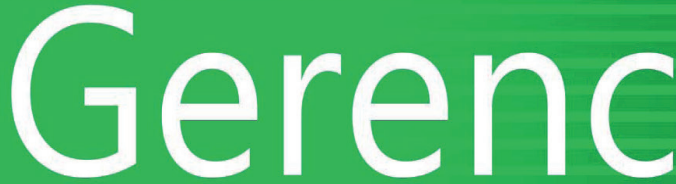

0

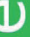

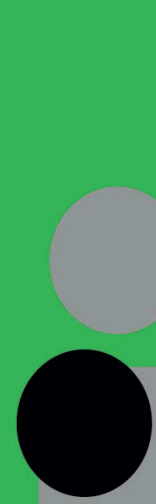

ro

u

$>$

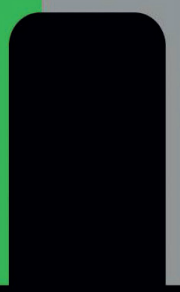




\title{
Desarrollo local sostenible de la ciudad de Cartagena de Indias, Colombia. Periodo 2010- 2016
}

\author{
Ramos Ríos, Marcela Sofía ${ }^{1}$ \\ Barrios Martinez, Alejandro ${ }^{2}$ \\ Vargas Atencio, Yuranis ${ }^{3}$ \\ Cifuentes Cifuentes, Omar ${ }^{4}$
}

\section{Resumen}

Los objetivos de Desarrollo Sostenible como metas de sostenibilidad al 2030, en su objetivo número 11 denominado Ciudades sostenibles, buscan lograr que las ciudades y los asentamientos humanos sean inclusivos, seguros, resilientes y sostenibles. Esta investigación tiene como objetivo analizar la sostenibilidad del desarrollo local de la ciudad de Cartagena Colombia, de manera que esto permita comprender la dinámica de la gestión del mismo en cada una de sus dimensiones. El estudio es de corte cuantitativo de tipo documental y transversal, soportado en fuentes secundarias, se estudian indicadores de las dimensiones económica, social, ambiental y de gestión pública en los periodos 2010 a 2016, bajo la metodología del biograma e índice de desarrollo sostenible (IDS). Los resultados presentan gráficamente el estado de sostenibilidad de la ciudad y un índice de desarrollo sostenible para los periodos estudiados, destacando un valor de 0,40 considerado crítico en el 2010 a un valor de 0,55 en el 2016, indicando que la dinámica de la gestión del desarrollo local sostenible de Cartagena es inestable en la dimensión social, ambiental y la gestión pública.

Palabras clave: Desarrollo local sostenible; sostenibilidad; Cartagena de Indias.

Recibido: 21-10-19 Aceptado: 10-11-19

Doctora en Ciencias Sociales Mención Gerencia Universidad del Zulia. Docente investigadora Fundación Universitaria Tecnológico Comfenalco -Cartagena Colombia. mramosr@tecnocomfenlaco.edu.co . https:// orcid.org/0000-0001-9277-9506

2 Candidato a Doctor en ciencias sociales Mención Gerencia Universidad del Zulia. Docente investigador Universidad de Cartagena Colombia. abarriosm@unicartagena.edu.co.

3 Candidata a Doctora en Economía y empresa universidad Castilla De La Mancha España .Docente investigadora Fundación Universitaria Tecnologico Comfenalco-Cartagena.yvargas@tecnocomfenalco.edu. co

4 Magister en Administración. Docente investigador Institución Tecnológica Colegio Mayor de Bolívar Colombia Cifuentes.omar@gmail.com 


\title{
Sustainable local development of the city of Cartagena de Indias Colombia. 2010-2016 periods
}

\begin{abstract}
The Sustainable Development goals as sustainability goals by 2030 , in its objective number 11 called Sustainable Cities, seek to make cities and human settlements inclusive, safe, resilient and sustainable. This research aims to carry out an analysis of the sustainability of the local development of the city of Cartagena Colombia, which allows understanding the dynamics of its management in each of its dimensions. The study is of quantitative cut of documentary and transversal type, supported in secondary sources, indicators of the economic, social, environmental and public management dimensions are studied in the periods 2010 to 2016 , under the methodology of the biogram and index of sustainable development. The results graphically show the state of sustainability of the city and an index of sustainable development for the periods studied, highlighting a value of 0.40 considered critical in 2010 to a value of 0.55 in 2016, indicating that the dynamics of Cartagena's sustainable local development management is unstable in the social, environmental and public management dimension.
\end{abstract}

Keywords: Sustainable local development; sustainability; Cartagena de Indias.

\section{Introducción}

Cartagena de Indias es la Capital del Departamento de Bolívar Colombia, fue fundada en 1533 por Don Pedro de Heredia, y de acuerdo con la Constitución Política de Colombia de 1991 es un Distrito Turístico, Histórico y Cultural, declarada Patrimonio Histórico y Cultural de la Humanidad por la UNESCO en 1985 (Alcaldia de Cartagena, 2015).

La ciudad de Cartagena, en el periodo estudiado 2010-2016, ha presentado una inestabilidad en su gobierno local, durante ese periodo de tiempo ha tenido 10 alcaldes $y$ ningún Plan de Desarrollo se ha ejecutado totalmente, a diferencia de otras ciudades donde los alcaldes han cumplido su periodo de 4 años para los que fueron elegidos y los planes de desarrollo han tenido una ejecución normal. La ciudad también presenta un caos cotidiano de movilidad, altos índices de pobreza y desigualdad, lo que se traduce en demandas de opciones de desarrollo sostenible que cada día deben ser analizadas.

Por ello se propuso como objetivo analizar la sostenibilidad del desarrollo local e la ciudad de Cartagena Colombia, que permita comprender la dinámica de la gestión del mismo en cada una de sus dimensiones que sirvan como referentes para el diseño de un modelo de gestión del desarrollo local orientado al logro 
de ciudades sostenibles que lleve a pensar en nuevas maneras de gestionar los asuntos públicos y colectivos de las ciudades.

Para analizar la dinámica del desarrollo sostenible de la ciudad se ha planteado un estudio de corte cuantitativo de tipo documental y transversal, soportado en fuentes secundarias, bajo la metodología del Biograma y el índice de Desarrollo Sostenible (IDS), se estudian indicadores de las dimensiones económica, social, ambiental y de gestión pública en los periodos 2010 a 2016 , este periodo se escogió teniendo en cuenta la crisis política y de gobernabilidad que ha afrontado la ciudad en ese periodo, con el constante cambio de Alcalde municipal que no ha permitido la continuidad de los planes de desarrollo.

Los fundamentos teóricos de la investigación están sustentados en los postulados alrededor del concepto de desarrollo local, desarrollo sostenible y ciudades sostenibles.

El Banco Mundial (2010), considera al "desarrollo local como el trabajo conjunto que desempeña una comunidad, con la finalidad de alcanzar un crecimiento económico sostenido, que permita beneficios económicos y mejoras en la calidad de vida de las personas que viven en esa comunidad,( Montaño (2014: 2).

En esa misma línea de pensamiento de autores como (Vázquez, A. 1999; Llorens, J. L., et al, 2002; Alburquerque, 2004), coinciden en definir el desarrollo económico local como un proceso estructural y de crecimiento que, mediante el máximo aprovechamiento de los recursos locales, permite que las personas que viven en un área local o una región de un país experimenten un incremento continuo de su bienestar y que el proceso de desarrollo local contiene tres dimensiones la económica, la sociocultural y la política administrativa, la primera incluye los medios de producción, para generar productividad, la segunda se refiere a la red social que apoya los procesos y la tercera a la institucionalidad que crea el entorno favorable al desarrollo local.

Por otro lado el Informe Brundtland, ONU 1987 referido por Ruiz (2014), sustenta que el desarrollo sostenible es un concepto relativo al interés público que busca el crecimiento económico y el uso de los recursos naturales a nivel mundial, pero teniendo muy en cuenta los aspectos medioambientales y sociales globales, para que en el largo plazo no se comprometa ni se degrade la vida en el planeta, ni la calidad de vida de la especie humana. "La insostenibilidad del desarrollo, es actualmente uno de los desafíos más apremiantes en el siglo XXI". (Yánez, R, \& Zavarce, C., 2011:90).

Plasencia Soler et al, (2018), sostienen que a nivel internacional, en los últimos años son varias las iniciativas de carácter mundial que promueven el desarrollo sostenible, tales como los Objetivos de Desarrollo del Milenio de las Naciones Unidas (ONU); el Plan de Implementación de la UNESCO denominado Década de la Educación para el Desarrollo Sostenible; los Objetivos de Desarrollo Sostenible del Programa de las Naciones Unidas para el Desarrollo (PNUD). Los Objetivos de Desarrollo Sostenible como metas de sostenibilidad al 2030 , en su objetivo número 11 denominado Ciudades sostenibles busca lograr que las ciudades y los asentamientos humanos sean inclusivos, seguros, resilientes y sostenibles.

Kuroiwa (2003), menciona que 
Desarrollo local sostenible de la ciudad de Cartagena de Indias, Colombia. Periodo 2010-2016

Ramos Ríos, Marcela Sofía; Barrios Martinez, Alejandro; Yuranis Vargas Atencio; Cifuentes Cifuentes, Omar

una "ciudad sostenible debe ser segura, ordenada, saludable, atractiva cultural y físicamente, eficiente en su funcionamiento y desarrollo sin afectar al medio ambiente natural, ni el patrimonio histórico cultural y, como consecuencia de todo ello, gobernable" (Londoño, 2011: 200).

En este sentido, el desarrollo local será sostenible, si contribuye al mejoramiento de la calidad de vida de sus habitantes es decir si los ciudadanos tienen la posibilidad de satisfacer las necesidades básicas alimentación, salud, educación, trabajo, seguridad, vivienda así como un medio ambiente de alta calidad en los alrededores, el desarrollo local debe estar orientado al manejo sostenible de los recursos territoriales sin degradarlos, ni descuidarlos para que sirvan a las generaciones futuras.

\section{Desarrollo local sostenible de Cartagena: Caracterización}

Para la caracterización del desarrollo sostenible, se tuvo en cuenta las dimensiones mencionadas por Sepúlveda (2008): la económica, social, ambiental y política administrativa, esta ultima los autores la llaman gestión pública (ilustración 1). Los Indicadores para cada dimensión se soportaron en los planteados por la Comisión en Desarrollo Sostenible de las Naciones Unidas mencionados por Arias (2006), adaptados a las condiciones de la ciudad de Cartagena.

\section{Ilustración 1 Dimensiones del desarrollo sostenible de un territorio}

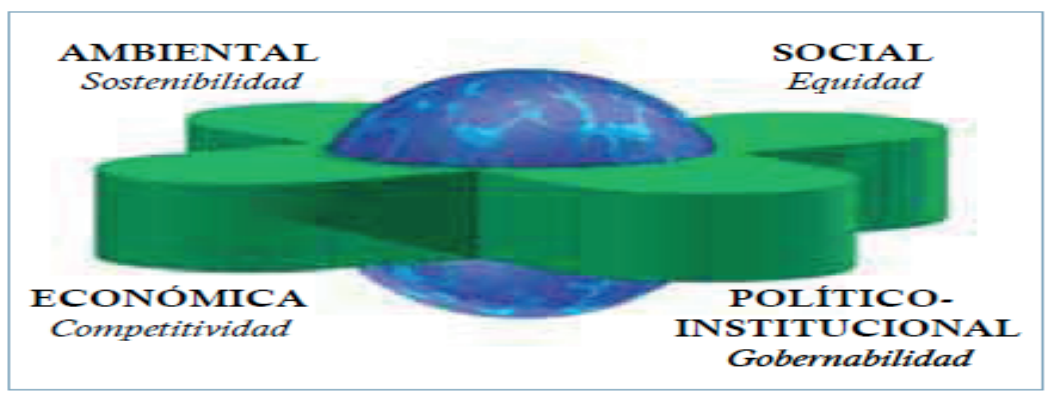

Fuente: Sepúlveda (2008)

2.1 Dimensión económica: esta dimensión hace referencia al potencial económico de la ciudad para generar los bienes y riquezas que promuevan la prosperidad o bienestar para el presente y futuro de los ciudadanos. Se revisó la contribución de los sectores a la generación de empleo, la tasa de ocupación, el índice de desempleo y la informalidad del empleo.

Cartagena tiene una vocación concentrada principalmente en el turismo, como una de las actividades más destacadas, sumado a la importancia de 
los sectores industrial y portuario (Pérez et al, 2011).

Según datos de Invest in Cartagena (2016), el sector de comercio, hoteles y restaurantes aporta el $32,4 \%$ del empleo de la ciudad, seguido del sector servicios comunales sociales y personales con el $22,8 \%$, el sector de transportes y comunicaciones constituye el $14,5 \%$ del empleo, el sector de la construcción aporta el 8,7\%.y la industria manufacturera acoge al $9,5 \%$ de la mano de obra. La industria se concentra casi exclusivamente en las actividades petroquímicas y de fabricación de cemento, que son el $85 \%$ de toda la producción industrial, con la refinería de Ecopetrol como principal centro productivo.

El puerto de Cartagena es clave en la actividad económica de la ciudad, es la primera zona portuaria de Colombia y el $4^{\circ}$ en Latinoamérica en movimiento de contenedores. Por Cartagena se maneja: Más del $60 \%$ de la carga contenerizada de comercio exterior en el país, más del $50 \%$ de los trámites de comercio exterior y $93 \%$ del tránsito internacional (Invest in Cartagena 2016).

\section{Tabla 1}

\section{Ranking de movimientos de contenedores}

\begin{tabular}{|c|c|c|c|}
\hline Ranking & Puerto/Port & Pafs/Country & 2015 (TEU) \\
\hline 1 & Santos & Brasil & $3,645,448$ \\
\hline 2 & Colón & Panamá & $3,577,427$ \\
\hline 3 & Balboa & Panamá & 3.294 .113 \\
\hline 4 & Cartagena & Colombia & $2,606,945$ \\
\hline 5 & Manzanillo & México & $2,458,135$ \\
\hline 6 & Callao & Perú & $1,900,444$ \\
\hline 7 & Guayaquil & Ecuador & $1,764,937$ \\
\hline 8 & Kingston & Jamaica & $1,653,272$ \\
\hline 9 & Buenos Aires & Argentina & $1,433,053$ \\
\hline 10 & Freeport & Bahamas & $1,400,000$ \\
\hline
\end{tabular}

Fuente: Invest in Cartagena con base en Cepal

La tasa de desempleo en cada periodo estudiado ha tenido un comportamiento similar a la tasa de
Colombia, en el 2016 fue de 9,0\% y Colombia 9,2\% (gráfico 1).

\section{Gráfico 1 \\ Tasa de desempleo Cartagena}

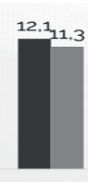

2008
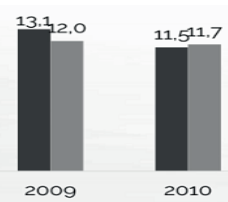

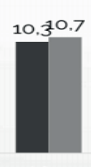

2011

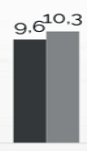

2012

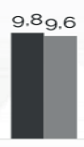

2013

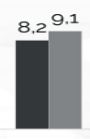

2014

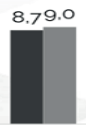

2015

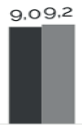

2016

Fuente: Cartagena Cómo Vamos (2016) 
Desarrollo local sostenible de la ciudad de Cartagena de Indias, Colombia. Periodo 2010-2016

Ramos Ríos, Marcela Sofía; Barrios Martinez, Alejandro; Yuranis Vargas Atencio; Cifuentes Cifuentes, Omar

La tasa de Informalidad del empleo desde el 2008 al 2017 ha estado por encima de la Colombia, alcanzando en el año $201654,4 \%$ y subiendo en el 2017 a 55,3\% (gráfico 2).

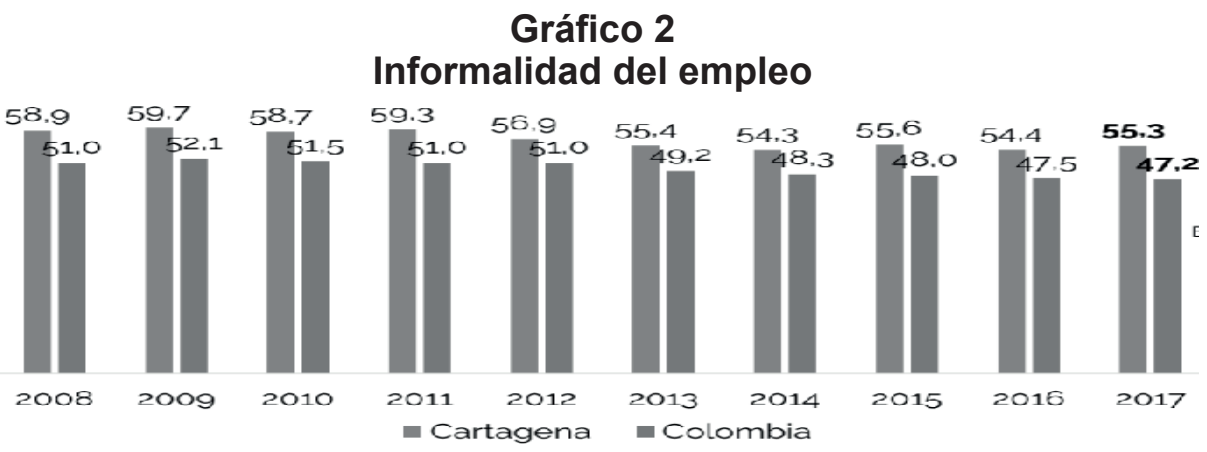

Fuente: DANE, elaboración Cartagena Cómo Vamos

2.2 Dimensión social: esta dimensión estudia la situación social y condiciones de vida básicas de los habitantes, se revisaron indicadores como el índice de pobreza monetaria, el índice de pobreza extrema, cobertura en educación, cobertura de servicios públicos y seguridad,
De acuerdo con Cartagena como vamos (2017), la situación de pobreza medida por ingreso a pesar de que ha venido disminuyendo desde 2008 en el 2016 fue de 29,1 y en el 2017 de $27 \%$ (gráfico 3).

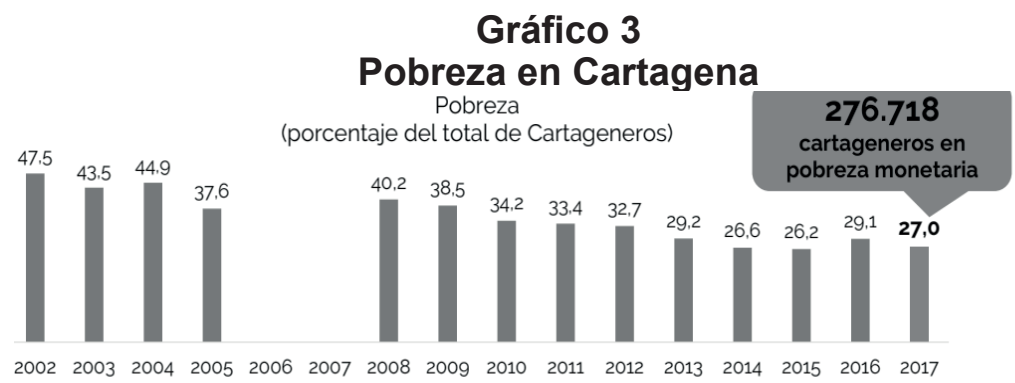

Fuente: Cartagena Cómo Vamos, encuesta calidad de vida 2017

El índice de pobreza extrema también ha venido disminuyendo con altos y bajos del 2008 cuando estaba en un $6,9 \%$ y al 2016 en $4,5 \%$ y 2017 se ubicó en 4,1\% (gráfico 4). 


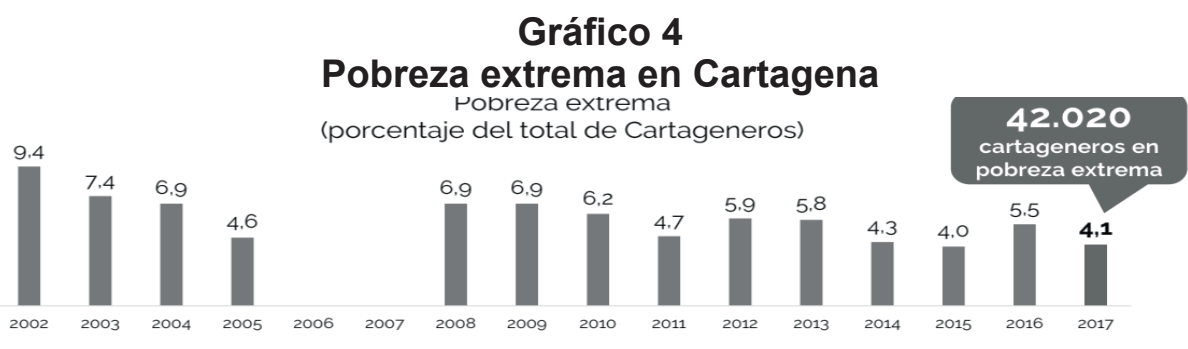

Fuente: Cartagena Cómo Vamos.

La cobertura en educación, en el nivel de preescolar que en el 2010 tenía un 65,9\% disminuyo en el 2016 presentando una cobertura de $64 \%$, en el nivel de secundaria tienen un $79 \%$ de cobertura neta, la primaria tiene con un $94 \%$ y la media técnica, viene en aumento llega a 2016 con el 51\% de cobertura neta, pero aún son bajas con respecto a otras ciudades del país. (Cartagena Cómo Vamos, 2017) (gráfico $5)$.

\section{Gráfico 5}

\section{Tasa de cobertura neta sin extra edad por niveles Cartagena

$$
\text { 口2010 } 2011 \quad 2012 \quad 2013 \quad 2014 \quad 2015 \square 2016
$$

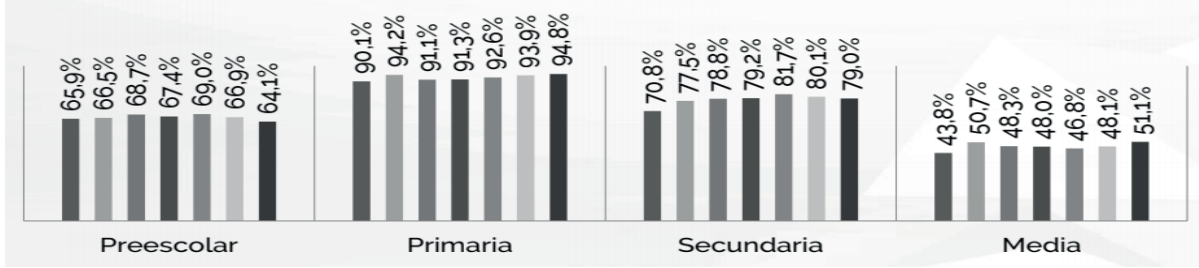

Fuente: Cartagena Cómo Vamos

En cuanto a los servicios públicos la ciudad tiene un tiene un $99,3 \%$ de cobertura en acueducto, y un e 93,6\%en alcantarillado (gráfico 6).

\section{Gráfico 6}

\section{Cobertura acueducto y alcantarillado Cartagena 2005-2016}

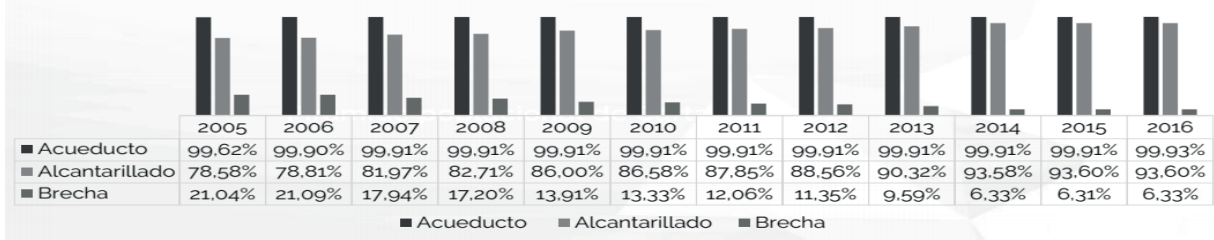

Fuente: Cartagena Cómo Vamos 
Desarrollo local sostenible de la ciudad de Cartagena de Indias, Colombia. Periodo 2010-2016

Ramos Ríos, Marcela Sofía; Barrios Martinez, Alejandro; Yuranis Vargas Atencio;

Cifuentes Cifuentes, Omar

En lo concerniente a la salud se puede constatar que el índice de mortalidad infantil en menores de un año por cada mil nacidos vivos ha venido disminuyendo desde 2010 que era de $16,4 \%$ a $11,5 \%$ en el 2016 y $10 \%$ en el 2017 (gráfico 7).

\section{Gráfico 7}

\section{Muertes en menores de 1 año por cada mil nacidos vivos}

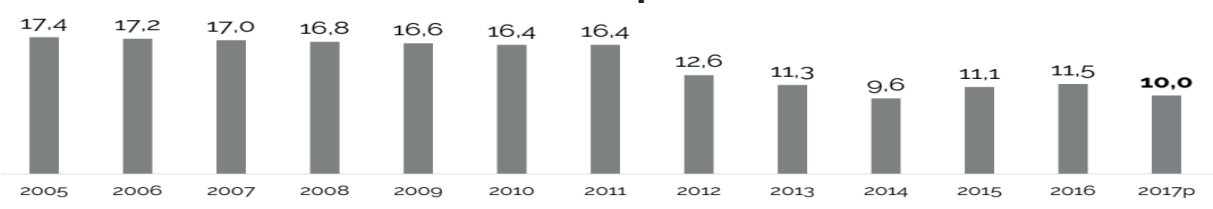

Fuente: Cartagena Cómo Vamos

El comportamiento de la seguridad en lo referente a muertes violentas en sus diferentes modalidades tiene cifras altas, en el 2010 tenía un $37,2 \%$ y en el 2016 un 43,4\% (gráfico 8).

\section{Gráfico 8 \\ Muertes violentas en Cartagena}

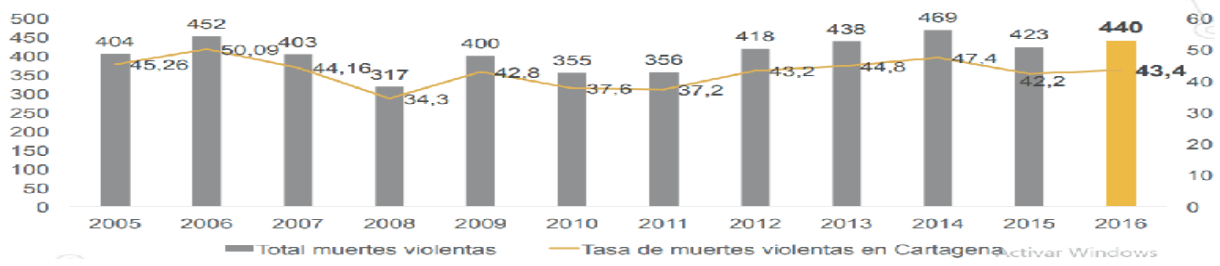

Fuente: COSED, Cartagena Cómo Vamos

2.3 Dimensión Ambiental: esta dimensión distingue al ambiente como base de la vida y por lo tanto, como fundamento del desarrollo sostenible. Por ello se revisaron indicadores que reflejan impactos en el ambiente, entre ellos, kgs de residuos sólidos por habitante y espacio público por habitante.

Los kilogramos de residuos sólidos diarios que producen los habitantes de Cartagena y las toneladas anuales, han ido en aumento desde el año 2013, (tabla 2). 


\section{Tabla 2 \\ Producción de residuos sólidos}

\begin{tabular}{|c|c|c|c|c|}
\hline RESIDUOS SÓLIDOS & $\mathbf{2 0 1 3}$ & $\mathbf{2 0 1 4}$ & $\mathbf{2 0 1 5}$ & $\mathbf{2 0 1 6}$ \\
\hline Toneladas producidas al año & 352.284 & 378.984 & 406.417 & 436.259 \\
\hline Kg diarios producidos por persona & 1,00 & 1,08 & 1,12 & 1,21 \\
\hline
\end{tabular}

Fuente: Cartagena Cómo Vamos 2017

En lo referido al espacio público, la Organización Mundial de la Salud (OMS) recomienda que la dotación mínima de espacio público efectiva por habitante debe ser de 15 metros cuadrados. La meta intermedia de Colombia es de 10 metros cuadrados, en Cartagena según los datos del Informe de Calidad de Vida para el año 2017 hay disponibles 4,72 metros cuadrados. Esto quiere decir, que Cartagena cuenta con un déficit de 10,28 metros cuadrados con relación a lo que dicta la OMS. Y el área urbana por metro cuadrado en el 2015 era de 0,62 (gráfico 11).

\section{Gráfico 11}

Espacio público por habitante

\begin{tabular}{|c|c|c|c|c|c|c|}
\hline \multicolumn{7}{|c|}{ Espacio Público por habitantes $\mathbf{M}^{2}$} \\
\hline & 11,63 & 11,5 & 11,36 & 11,23 & 11,09 & 10,96 \\
\hline 10 & 7,98 & 7,88 & 7,78 & 7,68 & 7,58 & 7,48 \\
\hline \multirow{2}{*}{5} & 4,75 & 4,69 & 4,63 & 4,57 & 4,51 & 4,45 \\
\hline & 0,62 & 0,61 & 0,61 & 0,6 & 0,59 & 0,62 \\
\hline \multirow{2}{*}{$\mathbf{o}$} & 2010 & 2011 & 2012 & 2013 & 2014 & 2015 \\
\hline & \multicolumn{2}{|c|}{ —Espacio público Total/Hab $(*)$} & $\begin{array}{l}\operatorname{lab}(*) \\
\operatorname{tab}(* *)\end{array}$ & \multicolumn{2}{|c|}{$\Longrightarrow$ Espacio público Urbano/Hab $\left(^{* *}\right)$} & $\because$ Parques Urbanos/Hab (**) \\
\hline
\end{tabular}

Fuente: Observatorio Ambiental de Cartagena

2.4. Política administrativa - gestión pública: esta dimensión permite mostrar la asignación de recursos y su redistribución entre diferentes actividades y grupos sociales y la transparencia de la información. Uno de los indicadores para mirar la gestión pública es el porcentaje de gastos de funcionamiento como proporción de ingresos corrientes de libre destinación
(ICDL), los cuales al terminar la vigencia 2016 tuvo un 43\%, lo que significa que está al límite del tope que establece la Ley 617 (Senado, 2000), dicha Ley estipula que al ser Cartagena Distrito Especial, el tope de ICLD dedicados a gasto de funcionamiento, debe ser máximo 50\%(Cartagena como vamos 2017) (gráfico 9). 


\section{Gráfico 9}

\section{Porcentaje de Gastos de Funcionamiento/ ICLD Cartagena}

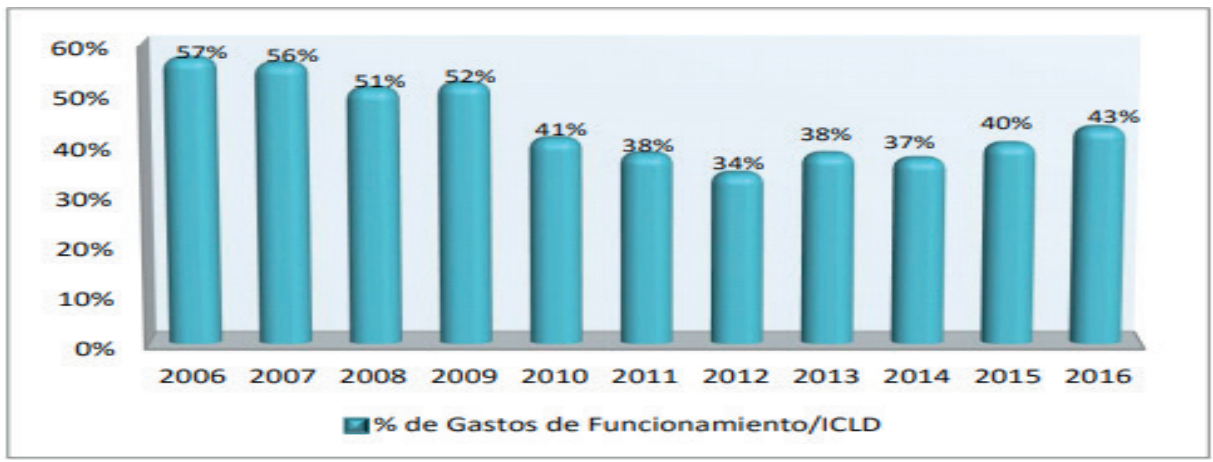

Fuente: Cartagena Cómo Vamos 2017

Otro indicador es el índice de desempeño fiscal que de conformidad con lo establecido en la Ley 617 de 2000, en Cartagena he mejorado a partir del
2010, manteniéndose en un promedio de $79 \%$. Que de acuerdo a los rangos establecidos es solvente (gráfico 10).

\section{Gráfico 10 \\ Índice de Desempeño Fiscal de Cartagena}

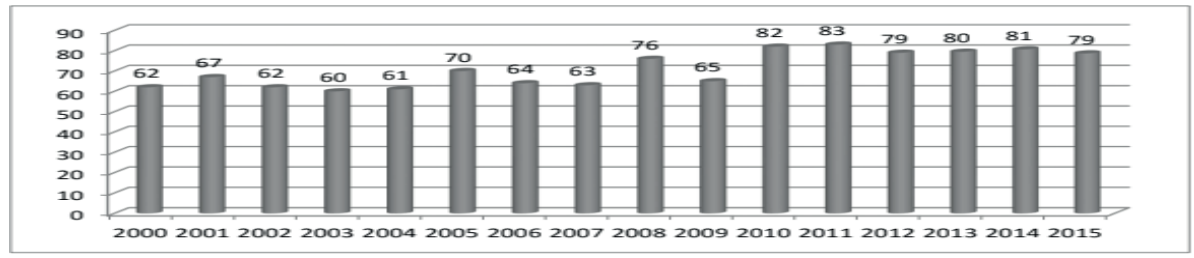

Fuente: Dane

El indicador que mide la transparencia en Colombia es el Índice de Gobierno Abierto (IGA), indica el grado de apertura con el reporte, tratamiento y divulgación de la información exigida por las entidades y normas de orden nacional en el 2016 Cartagena tiene un porcentaje de $60,8 \%$. Io que significa que el grado no llega a niveles altos de trasparencia en la información. 


\section{Metodología del Biograma y el índice de desarrollo sostenible: Aplicaciones esenciales}

\author{
El Biograma es un diagrama \\ multidimensional con imagen en \\ telaraña con sus índices respectivos que \\ representa gráficamente el estado de un \\ sistema. La imagen de telaraña muestra \\ el grado de desarrollo sostenible
}

del objeto de estudio. A través del Biograma se puede realizar un análisis comparativo de la evolución de las dimensiones del desarrollo sostenible en diferentes momentos de su historia. "Cada uno de los radios del círculo que componen el Biograma tiene un valor de 1, por lo que el valor de cada indicador individual variará entre 0 y 1 , siendo 0 el nivel mínimo y 1 el máximo" desempeño" (Sepúlveda 2008:5) (grafico 12).

\section{Gráfico 12 \\ Imagen de Biograma}

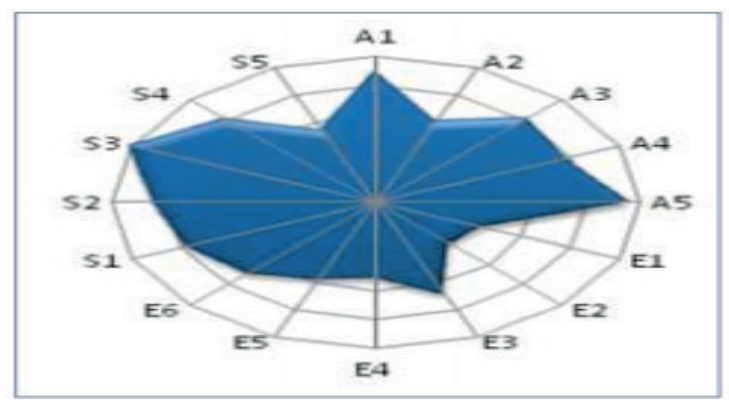

Fuente: Sepúlveda (2008)

En la imagen del Biograma se usan cinco colores para señalar el grado de desarrollo sostenible del territorio que se esté analizando. Cuando el área sombreada tiene un valor por debajo de 0.2 , se le coloca un color rojo que simboliza el estado del desarrollo con una alta probabilidad de colapso. Para valores entre 0.2 y 0.4 se usa el color anaranjado, para indicar un estado crítico. En valores de 0.4 a 0.6 el color utilizado es el amarillo, mostrando un sistema inestable y de 0.6 a 0.8 se maneja el color azul, indicando un sistema estable. De 0.8 a 1 el color es verde y se considera como la situación óptima del sistema (Sepulveda, 2008), (gráfico 13). 


\section{Gráfico 13 \\ El Estado del Sistema según los Colores del Biograma}

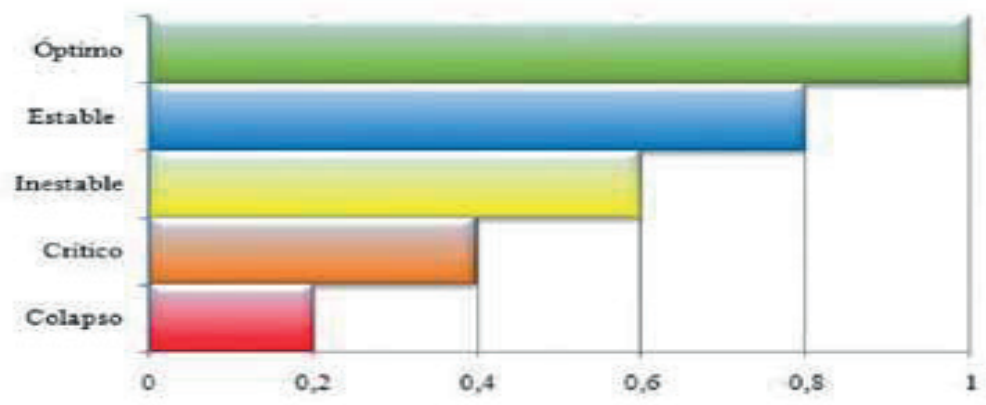

Fuente: Sepúlveda (2008)

Para generar la imagen del Biograma y del índice de desarrollo sostenible se definen las dimensiones económica, social, ambiental y gestión pública y los indicadores equivalentes a cada una de ellas, posteriormente se establecen los niveles máximos y mínimos de cada variable, de acuerdo a los datos observados, se relativizan los datos y por último se calcula el índice de desarrollo sostenible y la imagen del Biograma.

Para el análisis se seleccionaron cuatro dimensiones cada una con sus indicadores considerando la importancia representativa de cada uno en la gestión del desarrollo local sostenible de la ciudad.

3.1 Dimensión Social (DS): en esta dimensión se seleccionan como indicadores, el índice de pobreza monetaria, el índice de pobreza extrema, cobertura de servicios públicos, índice de mortalidad infantil en menores de cinco años y porcentaje de muertes violentas (tabla 3 ).

Tabla 3

Indicadores dimensión social

\begin{tabular}{cc}
\hline Indicador & Simbología \\
\hline Indice de pobreza monetaria & $\mathrm{S} 1$ \\
Indice de pobreza extrema & $\mathrm{S} 2$ \\
Cobertura de servicios públicos & $\mathrm{S} 3$ \\
Indice de mortalidad infantil menor de 5 años & $\mathrm{S} 3$ \\
Porcentaje de muertes violentas & $\mathrm{S} 4$
\end{tabular}

Fuente: elaboración propia 
En la tabla 4 se muestran los valores de los indicadores seleccionados expresados en porcentaje teniendo en cuenta los datos del diagnóstico de la ciudad.

\section{Tabla 4}

\section{Valores de Indicadores de la dimensión social}

\begin{tabular}{cccccc}
\hline Periodo & \multicolumn{5}{c}{ Dimensión Social } \\
& S1 & S2 & S3 & S4 & S5 \\
\hline 2010 & 34,2 & 6,2 & 99,1 & 16,4 & 37,6 \\
2011 & 33,4 & 4,7 & 99,1 & 16,4 & 37,2 \\
2012 & 32,7 & 5,9 & 99,1 & 14,8 & 43,2 \\
2013 & 29,2 & 5,8 & 99,1 & 14,1 & 44,8 \\
2014 & 26,6 & 4,3 & 99,1 & 11,2 & 47,4 \\
2015 & 26,2 & 4 & 99,1 & 11,1 & 42,2 \\
2016 & 29,1 & 5,5 & 99,3 & 11,5 & 43,4 \\
\hline
\end{tabular}

Fuente: elaboración propia

3.2 Dimensión Económica (DE): índice de precio al consumidor y tasa de para su análisis se incluye el índice Gini, $\quad$ informalidad del empleo (tabla 5 y 6 ) tasa de desempleo, tasa de ocupación,

\begin{tabular}{lc}
\multicolumn{2}{c}{$\begin{array}{c}\text { Tabla } 5 \\
\text { Indicadores dimensión Económica }\end{array}$} \\
\hline \multicolumn{1}{c}{ Indicador } & Simbología \\
\hline Indice Gini & E1 \\
Tasa de desempleo & E2 \\
Tasa de ocupación & E3 \\
Indice de precio al consumidor IPC & E4 \\
Tasa de informalidad del empleo & E5 \\
\hline
\end{tabular}

Fuente: elaboración propia. 
Desarrollo local sostenible de la ciudad de Cartagena de Indias, Colombia. Periodo 2010-2016

Ramos Ríos, Marcela Sofía; Barrios Martinez, Alejandro; Yuranis Vargas Atencio;

Cifuentes Cifuentes, Omar

\begin{tabular}{lccccc}
\multicolumn{7}{c}{ Tabla 6 } \\
Valores Indicadores de la dimensión económica \\
\hline \multirow{2}{*}{ Periodos } & E1 & E2 & E3 & E4 & E5 \\
\hline 2010 & 0,489 & 11,5 & 49,9 & 3,2 & 58,99 \\
2011 & 0,488 & 10,3 & 52,1 & 4,2 & 59,1 \\
2012 & 0,482 & 9,6 & 53,8 & 3 & 57,28 \\
2013 & 0,475 & 9,8 & 53,9 & 1,6 & 54,5 \\
2014 & 0,483 & 8,2 & 55,3 & 3,5 & 55,1 \\
2015 & 0,467 & 8,7 & 55 & 7,4 & 57,2 \\
2016 & 0,46 & 9 & 52,3 & 5,25 & 53 \\
\hline
\end{tabular}

Fuente: elaboración propia

3.3 Dimensión Ambiental (DA): Los indicadores utilizados para el análisis de esta dimensión son: consumo de agua por habitante mensual, toneladas de residuos sólidos al mes, superficie de áreas verdes por habitante, espacio público mts cuadrados por habitante y calidad del aire (tabla 7 y 8 ).

\section{Tabla 7}

Indicadores de la dimensión ambiental

\begin{tabular}{lc}
\hline \multicolumn{1}{c}{ Indicador } & Simbología \\
\hline Consumo de agua por habitante litros diarios & A1 \\
Área verde Mts cuadrados por habitante & A2 \\
Calidad del aire Partículas sostenidas totales PST & A3 \\
Toneladas de residuos sólidos al año & A4 \\
\hline
\end{tabular}

Fuente: elaboración propia 
pp. $19-38$

Revista Venezolana de Gerencia, Año 24 Número especial 2, 2019

\begin{tabular}{ccccc}
\multicolumn{5}{c}{ Tabla 8} \\
Valores de Indicadores de la dimensión ambien \\
\hline \multirow{5}{*}{ Periodo } & A1 & A2 & A3 & A4 \\
\hline 2010 & 92,2 & 0,62 & 0,7 & 0 \\
2011 & 92,2 & 0,61 & 0,7 & 0 \\
2012 & 101,7 & 0,61 & 0,7 & $2,888,9$ \\
2013 & 97,4 & 0,6 & 0,7 & 992,3 \\
2014 & 97,4 & 0,59 & 0,75 & 579 \\
2015 & 97,4 & 0,62 & 0,78 & 5216,6 \\
2016 & 97,4 & 0,62 & 0,78 & 5216,6 \\
\hline
\end{tabular}

Fuente: elaboración propia

3.4 Dimensión Pública (DP): ingresos corrientes de libre destinación para el análisis de esta dimensión se (ICDL), índice de gobierno abierto, incluyeron los indicadores porcentaje índice de desempeño fiscal y porcentaje de inversión, porcentaje de gastos de de respaldo a la deuda (Tabla 9 y 10).

funcionamiento como proporción de

Tabla 9

Indicadores de la Gestion publica

\begin{tabular}{lc}
\hline Indicador & Simbología \\
\hline Porcentaje de inversión & P1 \\
$\begin{array}{lc}\text { Porcentaje gastos de funcionamiento como propor- } \\
\text { ción ingresos corrientes de libre destinación ICDL }\end{array}$ & P2 \\
Indice de gobierno abierto & P3 \\
Indice de desempeño fiscal & P4 \\
Porcentaje respaldo de la deuda & P5 \\
\hline
\end{tabular}

Fuente: elaboración propia. 
Desarrollo local sostenible de la ciudad de Cartagena de Indias, Colombia. Periodo 2010-2016

Ramos Ríos, Marcela Sofía; Barrios Martinez, Alejandro; Yuranis Vargas Atencio;

Cifuentes Cifuentes, Omar

\section{Tabla 10}

Valores de los indicadores de la Gestión publica

\begin{tabular}{cccccc}
\hline \multirow{2}{*}{ Periodo } & \multicolumn{5}{c}{ Dimensión publica } \\
& P1 & P2 & P3 & P4 & P5 \\
\hline 2010 & 76 & 41 & 37,5 & 82 & 11 \\
2011 & 83 & 38 & 59,3 & 83 & 0,01 \\
2012 & 77 & 34 & 75,8 & 79 & 10,7 \\
2013 & 81 & 38 & 70,9 & 80 & 7,1 \\
2014 & 82 & 37 & 62,3 & 81 & 7,09 \\
2015 & 78 & 40 & 65,3 & 79 & 10,3 \\
2016 & 78 & 43 & 68 & 77 & 9,5 \\
\hline
\end{tabular}

Fuente: elaboración propia

\subsection{Calculo del Indice de desarrollo sostenible y elaboración del Biograma}

Siguiendo a Sepúlveda (2008:33), con el fin de adaptar los indicadores a una escala común, se utiliza una función de relativización.

1. Para el caso de los indicadores que presentan una relación positiva con el desarrollo sustentable, se empleó la siguiente: $F(x)=x-m / M-m$

2. Para el caso de los indicadores que presentan una relación negativa con el desarrollo sustentable, se empleó lo siguiente: $F(x)=x-M / m-M$

Al aplicar las formulas, los valores obtenidos oscilan entre 0 y 1 , y para ambos casos el valor 1 implica situación mejor, lo que indica que los datos se han estandarizado. Para el cálculo del índice de desarrollo sostenible, se utiliza una fórmula que primero calcula el promedio ponderado de los indicadores de cada una de las dimensiones, previamente relativizados. Después de calcular el promedio de cada dimensión, estas se ponderan de acuerdo al nivel de importancia estipulado por el usuario.

La fórmula para calcular el Índice de cada dimensión es la siguiente:

$$
S_{D}=\frac{7}{n_{D}} \sum_{i=1}^{n_{D}} I_{i}^{D}
$$

(Ecuación 1)

Fuente: Sepúlveda (2008)

En donde $\mathrm{S}$ es el indicador de la dimensión $D$ y se entiende que esa dimensión tiene $n D$ indicadores. Por tanto $S D$ es un promedio de los indicadores de la dimensión, los cuales han sido previamente estandarizados, para que tomen valores entre $0 \mathrm{y}$ 1. Luego los índices de todas las dimensiones se agregan para obtener el índice integrado. La agregación se 
hace ponderando cada dimensión por un porcentaje de importancia $(D)$ La fórmula para calcular el índice integrado de desarrollo sostenible es:

$$
S^{3}=\sum_{1}^{M}\left(\frac{\beta_{D}}{700}\right) S_{D}
$$

(Ecuación 2)

Fuente: Sepúlveda 2008
Dónde:

$\beta D$ : Porcentaje de importancia de cada dimensión.

SD: Promedio de los indicadores para cada dimensión.

S3: índice de sustentabilidad agregado.

Luego de haber estandarizados los datos de cada dimensión estudiada con sus respectivos indicadores se tienen los resultados del índice de desarrollo sostenible para cada año y por cada dimensión (tabla 11) y la imagen del Biograma (ilustración 2)

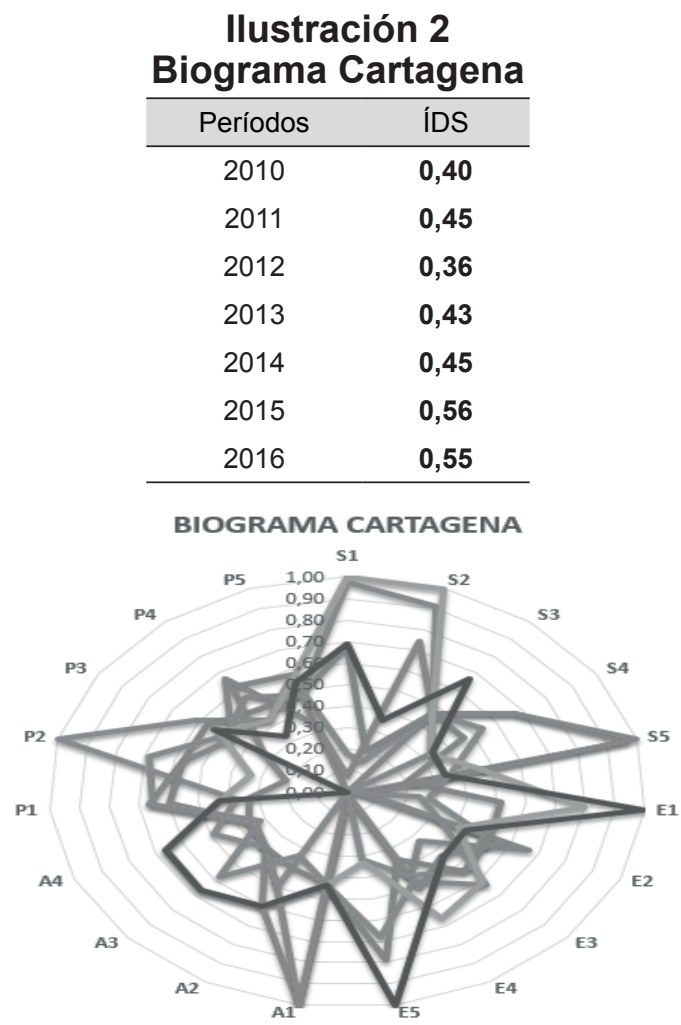

Fuente: elaboración propia basado en Sepúlveda (2008) 
Ramos Ríos, Marcela Sofía; Barrios Martinez, Alejandro; Yuranis Vargas Atencio;

Cifuentes Cifuentes, Omar

\section{Tabla 11}

Índice de Desarrollo sostenible por cada dimensión

\begin{tabular}{cccccccccc}
\hline PERIODO & $\begin{array}{c}\text { ÍNDICE } \\
\text { DS }\end{array}$ & B1 & $\begin{array}{c}\text { ÍNDICE } \\
\text { DE }\end{array}$ & B2 & $\begin{array}{c}\text { INDICE } \\
\text { DA }\end{array}$ & B3 & $\begin{array}{c}\text { INDICE } \\
\text { DP }\end{array}$ & B4 & $\begin{array}{c}\text { ÍNDICE } \\
\text { DESARROLLO } \\
\text { SOSTENIBLE }\end{array}$ \\
\hline 2010 & 0,42 & 0,30 & 0,30 & 0,30 & 0,58 & 0,20 & 0,34 & 0,20 & 0,40 \\
2011 & 0,59 & 0,30 & 0,32 & 0,30 & 0,56 & 0,20 & 0,34 & 0,20 & 0,45 \\
2012 & 0,34 & 0,30 & 0,41 & 0,30 & 0,34 & 0,20 & 0,31 & 0,20 & 0,36 \\
2013 & 0,40 & 0,30 & 0,54 & 0,30 & 0,39 & 0,20 & 0,35 & 0,20 & 0,43 \\
2014 & 0,54 & 0,30 & 0,48 & 0,30 & 0,42 & 0,20 & 0,34 & 0,20 & 0,45 \\
2015 & 0,65 & 0,30 & 0,56 & 0,30 & 0,59 & 0,20 & 0,39 & 0,20 & 0,56 \\
2016 & 0,48 & 0,30 & 0,69 & 0,30 & 0,59 & 0,20 & 0,42 & 0,20 & 0,55 \\
\hline
\end{tabular}

Fuente: elaboración propia

El Biograma de Cartagena y el Índice de Desarrollo sostenible para el período estudiado, permite analizar que aunque ha venido en aumento el índice de desarrollo sostenible al crecer por encima de 0.40 , a partir de 2010, los resultados de las dimensiones analizadas están por debajo de 0.60 , siendo considerada como inestables.

Al desagregar los índices por cada dimensión se obtuvo que la dimensión que más evolucionó durante estos periodos en la ciudad de Cartagena fue la Económica (DE) que pasó de un índice de 0,30 en el 2010 considerado crítico a un índice de 0.69 , en el 2016 considerado estable. Aunque se destaca también, los grandes esfuerzos realizados en la dimensión ambiental (DA) que casi alcanza como resultado un índice de 0.60. Caso contrario se observa en los indicadores de las dimensiones sociales (DS) y gestión pública (GP), los cuales resultaron evaluados como inestables, ya que si bien presentan valores por encima de 0.40 , no superan los 0,60 . Esto se debe específicamente a las pocas inversiones en proyectos sociales que impactan y trasciendan en la comunidad, la deuda fiscal y el porcentaje de respaldo de la misma; todo ello se traduce en el incremento de la pobreza extrema y monetaria, además, la falta de empleos que dispara la tasa de informalidad, abonados también a los comportamientos inestable en la gestión del gasto y transparencia de la información ya que la ciudad ha estado permeada por la corrupción.

\section{Conclusiones}

La dinámica de la gestión del desarrollo local sostenible de Cartagena muestra la realidad social y económica de la misma, la pobreza, la inequidad y desigualdad son el resultado de la confluencia de procesos y acciones concretas definidos por la ineficiente gestión pública reflejada en la crisis institucional que ha vivido la ciudad.

Analizar la dinámica del desarrollo local sostenible de la ciudad en varios periodos puede servir como punto de referencia a la administración local para el abordaje de proyectos que orienten la trasformación de la ciudad hacia la concreción de elementos de bienestar y factores de progreso para la ciudadanía. La gestión pública debe buscar de 
manera eficaz y eficiente resultados frente a la reducción de la pobreza y el mejoramiento de la calidad de vida de sus habitantes.

\section{Referencias bibliográficas}

Alburquerque, Francisco (2004), Desarrollo económico local y descentralización en América Latina, Revista de la CEPAL, 157.

Alcaldia de Cartagena (2015), Información Cartagena, Recuperado en Abril de 2017, de www.cartagena. gov.co.

Arias, Fabio (2006). Desarrollo sostenible y sus indicadores, Revista Sociedad y Economía ISSN: 1657-6357 Universidad del Valle Colombia, pp 22-224. Disponible en: http://www.redalyc.org/articulo. oa?id=99616177008

Banco Mundial, (2010), Desarrollo y cambio climático, Panorama general. Un nuevo clima para el desarrollo. Washington. Estados Unidos.

\section{Cartagena}

Como Vamos(2017),Encuesta Calidad de Vida, Recuperado en mayo de 2018, de http://www.cartagenacomovamos. org/nuevo/.

Invest in Cartagena (2016), Sectores de inversión. Recuperado el 2017, de http/www.investincartagena.com/ sectores-de-inversion.

Kuroiwa, Ikuo (2003), La gobernabilidad en los paises en desarrollo, En $\mathrm{K}$. Ikuo. International economic study group

Londoño, Linares, Juan Pablo (2011). Modelización de problemas ambientales en entornos urbanos Susceptibilidad a Deslizamientos de Tierra en Ciudades Andinas p200.recuperadodehttps://is.upc. edu/ca/documents/doctorat/tesisjuanplondonol.pdf

Llorens, Juan, Alburquerque, Francisco \& Del Castillo, Jaime (2002), Estudios de casos de desarrollo económico local en América Latina. InterAmerican Development Bank. Washington, D. C

Montaño,Maria Angelica (2014), Modelo de desarrollo económico local para la diversificación de la estructura productiva y la articulación del tejido empresarial de Baja California. (Tesis Doctoral). Eumed.net, p2.

ODS (2030), Objetivos del sedarrollo sostenible programa de las naciones unidas para el desarrollo. Recuperado el 2017, de https/www. und:org/content/undp/es/home/ sustainable-development-goasls. html.

Pérez, Dewin, Mercado, Menis, Quejada, Raúl (2011). Diagnóstico socioeconómico y del mercado de trabajo Cartagena de indias Recuperado el 2017,dehttp:// hacienda.cartagena.gov.co/ imagees/papers/Diagnósticosocioeconómico-y-del-mercado-detrabajo.pdf.

Placencia, Juan, Marrero, Fernando, Bajo Sanjuán Anna María, Nicando Miriam(2018), Modelos para evaluar la sostenibilidad de las organizaciones. Estudios Gerenciales, vol. 34, N 146, P 64

Procuraduría De La Nación (2017), Índice de Gobierno Abierto (IGA) https://www.procuraduria.gov.co/ portal/Indice-de-Gobierno-Abierto. page. Recuperado 10 - 06 - 2017

Ruiz, Walther, (2014), El plan de desarrollo urbano como instrumento técnico y político. Recuperado en 2017, de ocplayer. 
Desarrollo local sostenible de la ciudad de Cartagena de Indias, Colombia. Periodo 2010-2016

Ramos Ríos, Marcela Sofía; Barrios Martinez, Alejandro; Yuranis Vargas Atencio;

Cifuentes Cifuentes, Omar

es/21740038-El -plan-de-desarrollourbano.html.

Senado,(2000), Ley orgánica de presupesto 617, Recuperado el 2017, de http:www.secretariasenado. gov.co/s en a do/bas edoc/ ley-0617-2000.html.

Sepulveda,Sergio, (2008), Metodologia para estimar el nivel de desarrollo sostenible de territorios: biograma 2008. San Jose de Costa Rica: Instituto Interamericano para Agricultura ICA.
Vázquez Barquero, Antonio, (1999). Desarrollo, redes e innovación. Lecciones sobre desarrollo endógeno. Pirámide, Madrid.

Yánez, Raiza, Zavarce, Carlos (2011), Desarrollo sustentable y la investigación científica en las universidades autónomas venezolanas: retos y contradicciones. Revista Venezolana de Gerencia, No53, p 90 .

- Esta obra está bajo una licencia de Creative Commons Reconocimiento-NoComercialCompartirlgual 3.0 Unported. http://creativecommons.org/licenses/by-nc-sa/3.0/deed.es_ES 